\title{
A Genomically Characterized Collection of High-Grade Serous Ovarian Cancer Xenografts for Preclinical Testing
}

\author{
Paulina Cybulska, ${ }^{* \dagger}$ Jocelyn M. Stewart, ${ }^{\ddagger \S}$ Azin Sayad, ${ }^{\ddagger}$ Carl Virtanen,,${ }^{\ddagger}$ Patricia A. Shaw, ${ }^{\llbracket}$ Blaise Clarke, ${ }^{\llbracket}$ Natalie Stickle,
} Marcus Q. Bernardini, $* \star\|\|$ and Benjamin G. Nee ${ }^{\ddagger \xi * *}$

From the Departments of Obstetrics and Gynecology* and Medical Biophysics, ${ }^{\S}$ University of Toronto, Toronto, Ontario, Canada; the Department of Gynecologic Oncology, ${ }^{\dagger}$ Memorial Sloan Kettering Cancer Center, New York, New York; the Princess Margaret Cancer Centre $e^{\ddagger}$ and the Departments of Pathology" and Gynecologic Oncology, "University Health Network, Toronto, Ontario, Canada; and the Laura and Isaac Perlmutter Cancer Center, ** New York University Langone Medical Center, New York, New York

Accepted for publication January 16, 2018.

Address correspondence to Benjamin G. Neel, M.D., Ph.D. 522 First Ave., Smilow Bldg., Twelfth Floor, Ste. 1201, New York, NY 10016. E-mail: benjamin.neel@nyumc.org.

\begin{abstract}
High-grade serous ovarian cancer (HGSC) is the leading cause of morbidity and mortality from gynecologic malignant tumors. Overall survival remains low because of the nearly ubiquitous emergence of platinum resistance and the paucity of effective next-line treatments. Current cell culture-based models show limited similarity to HGSC and are therefore unreliable predictive models for preclinical evaluation of investigational drugs. This deficiency could help explain the low overall rate of successful drug development and the decades of largely unchanged approaches to HGSC treatment. We used gene expression, copy number variation, and exome sequencing analyses to credential HGSC patient-derived xenografts (PDXs) as effective preclinical models that recapitulate the features of human HGSC. Mice bearing PDXs were also treated with standard-of-care carboplatin therapy. PDXs showed similar sensitivity to carboplatin as the patient's tumor at the time of sampling. PDXs also recapitulated the diversity of genomic alterations (copy number variation and mutation profiles) previously described in large data sets that profiled HGSC. Furthermore, mRNA profiling showed that the PDXs represent all HGSC subtypes with the exception of the immunoreactive group. Credentialing of PDX models of HGSC should aid progress in HGSC research by providing improved preclinical models of HGSC that can be used to test novel targets and more accurately evaluate their likelihood of success. (Am J Pathol 2018, 188: 1120-1131; https://doi.org/ 10.1016/j.ajpath.2018.01.019)
\end{abstract}

High-grade serous ovarian cancer (HGSC) is the leading cause of morbidity and mortality from gynecologic malignant tumors. ${ }^{1,2}$ Typically, patients with this disease are treated with primary debulking surgery, followed by firstline platinum- and taxane-based chemotherapy, a regimen that has remained largely unchanged for several decades. Other patients receive neoadjuvant chemotherapy, followed by debulking. ${ }^{3-5}$ Overall survival remains low ${ }^{6}$ because of the nearly ubiquitous emergence of platinum resistance ${ }^{7,8}$ and the paucity of effective next-line treatments in platinum-resistant patients. ${ }^{9}$ Selection of second-line agents in platinum-resistant patients is typically physician dependent, mirroring the regimens used in the Avastin Use in Platinum-Resistant Epithelial Ovarian Cancer study if the
Supported by Canadian Institutes of Health Research grant CIHR-MOP191992, Canadian Cancer Society Research Institute-Terry Fox Program Project grant 020003, and the Samuel Waxman Cancer Research Foundation (B.G.N.). B.G.N. was a Canada Research Chair, Tier 1, and work in his Toronto laboratory was partially supported by a grant from the Ontario Ministry of Health and Long Term Care and the Princess Margaret Cancer Foundation. J.M.S. was supported by scholarship fund CIHR-CGS200710MDR from the Canadian Institutes for Health Research. P.C. was funded by a J.W. Knox Ritchie Research Award, the Kristi Piia Callum Memorial Fund in Ovarian Cancer Research, the Teresina Florio Graduate Scholarship in Cancer Research, and a Thomas G. Ryley Clinical Investigators Award.

P.C. and J.M.S. contributed equally to this work.

Disclosures: None declared. 
patient has platinum-resistant disease, or carboplatin paired with paclitaxel, gemcitabine, or liposomal doxorubicin in patients with platinum-sensitive disease. ${ }^{10}$ Aside from the use of poly (ADP-ribose) polymerase inhibitors for patients with $B R C A 1 / 2$ mutations, ${ }^{11}$ no validated biomarkers are available to rationally guide second-line therapy. Improved outcomes for patients with HGSC will require the development of more effective treatment options and the stratification of patients to appropriate therapeutics soon after diagnosis or on emergence of platinum resistance.

Large-scale genomic analyses of HGSC indicate substantial intertumor heterogeneity. Tothill et al ${ }^{12}$ examined 246 serous cancers (some of which were low-grade tumors) and, based on mRNA expression profiling, reported that these tumors could be classified into five subtypes. The Cancer Genome Atlas (TCGA) examined gene expression profiles, as well as copy number alteration (CNA), miRNA expression, and exome sequences for 489 HGSCs. This study reported that HGSC falls into four phenotypic subtypes: immune, mesenchymal, proliferative, and differentiated $^{13}$; however, many patients' tumors had features of more than one subtype. Neither study found significant differences in progression-free or overall survival in any unsupervised clustering-defined subgroup, which might reflect the poor overall prognosis, the lack of targeted therapeutics, and the relative absence of personalized approaches. Analysis by TCGA ${ }^{13}$ and subsequent studies by Verhaak et $\mathrm{al}^{14}$ demonstrated that, with supervised analysis, a gene expression signature of good and poor prognosis groups could be identified. Although this signature could provide useful prognostic information for patients with HGSC, it clearly requires prospective validation before use for patient stratification. Such stratification is necessary to identify patients with HGSC who might benefit from novel, targeted, and potentially subgroup-specific therapeutics, as well as for the discovery and development of such treatments.

Most studies of ovarian carcinogenesis and drug response have used immortalized cell lines grown for long periods in serum-containing culture. Whether these cells represent the biology of HGSC is questionable; indeed, a previous report indicates that several cell lines previously reported to be serous ovarian cancer in origin, including those used most frequently, do not show the genomic characteristics of HGSC. ${ }^{15}$ Many HGSC cell lines fail to reproduce serous histology when propagated as xenografts; others do not even yield xenografts. ${ }^{16}$ In culture, few lines show evidence of the cellular heterogeneity typically seen in primary tumors. ${ }^{15}$ Consequently, cell lines are unlikely to be reliable predictive models for preclinical evaluation of investigational drugs, which could help explain the low overall rate of successful drug development and the decades of largely unchanged approaches for the treatment of HGSC.

Well-characterized systems that reflect the genetic and cellular diversity of human HGSC more faithfully than conventional ovarian cancer cell lines and available genetically engineered mouse models could prove quite useful for drug development. Patient-derived xenografts (PDXs) provide a potential means to indefinitely propagate tumors that more closely represent human cancer. In this study, we describe our efforts to credential HGSC PDXs as effective preclinical models that recapitulate the features of human HGSC, using genomic and pharmacologic response criteria.

\section{Materials and Methods}

\section{Tumor Samples, Cells, PDX Establishment, and DNA Extraction}

Tumor cells were collected from fresh surgical material or ascites, as described previously. ${ }^{17}$ Notably, some PDXs studied here overlap with those used in the previous study (2028, 2140, 2194, 2345, 2489. 2555, 2685, 2753, 2803, $3224,3444,3352,3529,3635,3670,3748,4010$, and 4070). ${ }^{17}$ Briefly, HGSC tissue samples were obtained from the University Health Network Tissue Bank with patient consent and research ethics board approval. Histologic diagnosis and tumor content of all samples were verified by a gynecologic pathologist (P.A.S., B.C.). In addition, the histology of PDXs was compared with that of the primary tumor to show morphologic recapitulation of the primary tumor. Patient characteristics of samples used in this study are outlined in Supplemental Table S1. Tumors were procured within 2 to 4 hours of excision, minced, and digested with collagenase/hyaluronidase (Stem Cell Technologies, Vancouver, BC, Canada) in Dulbecco's modified Eagle's medium at $37^{\circ} \mathrm{C}$ for 2 hours. Red blood cells were lysed in $0.16 \mathrm{~mol} / \mathrm{L}$ ammonium chloride, and the remaining cells were filtered through a $70-\mu \mathrm{m}$ mesh and counted. Ascites cells were collected by centrifugation at $300 \times g$ and processed as above. Anti-CD45 microbeads were used to deplete leukocytes, as per the manufacturer's instructions (Miltenyi Biotec, Auburn, CA). For PDX establishment, CD45-depleted cells $\left(10^{5}\right.$ to $\left.10^{6}\right)$ in 1:1 HBSS/growth factor-reduced (dialyzed) Matrigel (BD Biosciences, San Jose, CA) were injected into the mammary fat pads of nonobese diabetic (NOD) and severe combined immunodeficiency (SCID) or NOD, SCID, and IL2 receptor $\gamma$-chain null (Il2rg) (NSG) mice, as previously described. ${ }^{17}$ Initially, PDXs were established in NOD/SCID mice, but when NSG became available, all remaining PDXs were established in these mice because, in general, tumor-initiating frequency is higher in this strain. All mouse studies were approved by the animal care committee at the University Health Network. Snap-frozen samples were obtained from the Neel Laboratory's PDX bank, and small samples were shaved from each frozen specimen for DNA extraction.

\section{PDX Response to Carboplatin}

Patient samples were identified retrospectively as platinum resistant $(3654,3875,6447,2555,2028,2462,2803,2489$, 
$3444,2903,6259,2685,2753,2261,4070,62143,6259)$ or platinum sensitive $(3748,3670,61382,63867,3028,3336)$ at the time of acquisition. All samples were required to have sufficient clinical information and to have been viably frozen in ample quantities to establish early passage $(1,2$, or 3) PDXs for treatment [3654 p2 (passage 2), $3875 \mathrm{p} 1,6447$ p0, 2555 p2, 2028 p2, 2462 p0, 2803 p2, 2489 p2, 3444 p1, 2903p1, 2685 p2, 2753 p3, 61382 p0]. Two PDX samples (3748 p2 and 67199 p0) were from tumors for which the genomic information was not available. Prospective samples (67199 p0, 67326 p0) were chemotherapy-naive, primary samples from patients followed up at the Princess Margaret Cancer Centre. CD45-depleted cells $\left(10^{6}\right)$ in 1:1 HBSS/ growth factor-reduced Matrigel (BD Biosciences) were injected into the mammary fat pads (one per mouse) of healthy, 6- to 8-week-old, female NSG mice. The maximum number of mice were injected with one vial of frozen cells; each patient sample had at least four mice per treatment arm (median, 5; range, 4 to 13 ) and $\geq 5$ mice in the control arm, cell number permitting. Once tumors reached $0.5 \mathrm{~cm}$, carboplatin $(10 \mathrm{mg} / \mathrm{mL}$; Hospira, Saint-Laurent, QC, Canada) was administered once weekly for a total of two doses. On the basis of the mouse-to-human drug translation formula ${ }^{18}$ and the assumption of a mean human adult weight of $60 \mathrm{~kg}$, the carboplatin dose for mice is approximately $125 \mathrm{mg} / \mathrm{kg}$. However, given the published carboplatin LD10 (dose at which $10 \%$ will die) of $100 \mathrm{mg} /$ $\mathrm{kg}$, published work using smaller $(<125 \mathrm{mg} / \mathrm{kg})$ doses, and our own MTD study, $75 \mathrm{mg} / \mathrm{kg}$ was determined as a more appropriate dose. ${ }^{7,8,19-21}$ Treatment mice received a total volume of $0.35 \mathrm{~mL}$ intraperitoneally $(75 \mathrm{mg} / \mathrm{kg}$ of carboplatin plus saline). Control mice received $0.35 \mathrm{~mL}$ of normal saline intraperitoneally. Tumors were measured using calipers for up to 1 week after the duration of treatment (total of 14 to 15 days after the first treatment).

\section{Sequencing and Mutation Calling}

Samples for DNA extraction included all 38 PDXs, 15 of which also had matched normal samples available. DNA was prepared by using proteinase $\mathrm{K}$ digestion, followed by phenol/chloroform extraction. DNA was sequenced on two lanes on an Illumina Hi-Seq 2000 (Illumina, San Diego, CA) using the targeted gene panel. Genes selected for analysis are the most commonly mutated genes in breast and ovarian cancer, according to the Catalogue of Somatic Mutations in Cancer (COSMIC) database (Sanger Institute, Cambridgeshire, UK; http://cancer.sanger.ac.uk/cosmic). Sample and library preparation, as well as sequencing, was performed as per the manufacturers' standard protocols. FASTQ files were generated using CASAVA software version 1.8.2 (Illumina), and overall quality of the run was checked using the FASTQC software package version 0.10.1 (Babraham Bioinformatics, Cambridge, UK). To reduce false-positive results in variant calling, all reads were classified as belonging to the mouse (mm10) or to the human (hg19) reference genomes using Xenome version 1.0.1. ${ }^{22}$ Human reads were aligned to the hg19 reference genome using Burrows-Wheeler Aligner software version $0.7 .2,{ }^{23}$ followed by removal of duplicates, indel realignment, and base quality score recalibration. For paired samples, realignment and base quality score recalibration models were built using tumor and normal data. Manual review of the TP53 locus in nonmutated samples (Supplemental Table S2) showed additional deletions, splicing variants, and frameshift mutations, with only three PDX demonstrating no variants in TP53.

\section{Somatic and PDX Variant Calling}

Variants in each sample were called using the software tool MuTect version 1.1.4 (Broad Institute, Cambridge, MA) with all default settings, except that the logarithm of odds score threshold for calling tumor variants was set to 15.0 , and the threshold for maximum alternate allele counts in normal samples was set to 10. Haplotype Caller from GATK version 3.0-0 (Broad Institute) was used to jointly call variants in the unpaired PDXs and the normal tissue samples as a separate group using default settings. Variants from Haplotype Caller were kept if they met the following criteria: approximate read depth $>10$, quality by depth $>2$, Records Management System mapping quality $>30$, and a phred-scaled $P$ value for the Fisher exact test to detect strand bias $<80$. Variants were annotated with Annovar and then filtered to remove those that were not exonic and/or were synonymous. ${ }^{24}$ These variants were then filtered further to remove those without any Cosmic-68 annotation or ClinVar annotation as pathogenic. If a gene was found to have a mutation that matched these criteria in at least two samples, it was retained, and this list was plotted using $\mathrm{R}$ version 3.0.2 ( $\mathrm{R}$ Foundation for Statistical Computing, Vienna, Austria). A subset of genes (TP53, BRCA1, BRCA2, CSMD3, NF1, RB1, FAT3, CDK12, $G A B R A 6$ ) that were expected to be mutated at a detectable frequency were analyzed manually to assess cutoff stringency, and these data were compared with the mutation frequencies reported by TCGA using $\chi^{2}$ analysis.

\section{Germline Variants}

Haplotype Caller from GATK version 3.0-0 was used to jointly call variants in the unpaired PDXs and the normal tissue samples as a separate group using default settings. Variants from Haplotype Caller were kept if they met the following criteria: approximate read depth $>10$, quality by depth $>2$, Records Management System mapping quality $>30$, and a phred-scaled $P$ value for the Fisher exact test to detect strand bias $<80$. Remaining variants were annotated with Annovar, and only those annotated as pathogenic by Clinvar and were nonsynonymous mutations with a frequency $<0.2$ were kept. Each of these mutations was verified in the Integrated Genomics Brower, version 2.3.40 (Broad Institute). 


\section{Illumina Single-Nucleotide Polymorphism Arrays}

Tumor and normal female control (Biochain lot B502039) genomic DNA samples (750 ng each) were amplified with an Illumina Infinium Genotyping multiuse kit. Amplified DNA was fragmented and precipitated, and one-third of the DNA was hybridized to 10 Human Omni-Quad Beadchips (Illumina). BeadChips were incubated at $48^{\circ} \mathrm{C}$ and hybridized for 17.5 hours. The BeadChips were washed and stained as per the Illumina protocol and scanned on the iScan (Illumina). Data files were quantified in GenomeStudio version 2011.1 (Illumina) using Omni-Quad Multiuse_H manifest (released April 2011), containing data from Genome Build 37 (hg19).

\section{Copy Number Segmentation of Single-Nucleotide Polymorphism Data}

Xenograft single-nucleotide data were segmented using the circular binary segmentation algorithm, implemented in the Bioconductor DNACopy package version 1.50 (Bioconductor, Buffalo, NY) with the following parameters: 1000 permutations, $\alpha=0.01$, and the merging of adjacent copy number segments for which the mean log-R ratio (LRR) differs by $<1 \mathrm{SD}$.

\section{Mapping CNA Segments to Genes}

Copy number segments that result from the circular binary segmentation algorithm were mapped to hg19 gene coordinates by using the Bioconductor CNTools package version 1.32 (Bioconductor). Gene coordinates were obtained from the University of California, Santa Cruz Genome Browser's table browser (https://genome.ucsc.edu/cgi-bin/ hgTables). ${ }^{25,26}$ For genes associated with multiple transcripts, the transcript associated with the longest transcription start-to-end length was taken as representative.

\section{Identification and Filtering of Genes Frequently Affected by Germline CNAs}

TCGA serous ovarian CNA segment data mapped to hg19 were downloaded from the Broad Institute's Firehose pipeline. Data with and without removal of germline CNAs deduced from matched normal samples were obtained. Segmented TCGA data were mapped to genes as described, yielding 24,584 (not germline CNA corrected) and 23,803 (germline corrected) mapped genes with CNA LRR values. The 781 genes absent from the corrected data set were assumed to be primarily attributable to the correction and were removed from the ovarian PDX data. A direct gene-to-gene comparison of the TCGA data with and without germline CNA correction showed an extremely close association (Pearson $r=0.995$ ), with a few outlier genes for which the LRR signals between the two data sets diverged visibly from the linear association. These outliers are likely to be genes for
Table 1 Patient Characteristics

\begin{tabular}{lrlr}
\hline Characteristic & Primary & Neoadjuvant & Recurrence \\
\hline Total & $18(47)$ & $3(8)$ & $17(45)$ \\
Sample source & & & \\
$\quad$ Ascites & $3(20)$ & $1(7)$ & $11(73)$ \\
$\quad$ Solid tumor & $15(65)$ & $2(9)$ & $6(26)$ \\
Age, years & & & \\
$\quad$ Median & 60 & 77 & 59 \\
$\quad$ Range & $44-76$ & $72-83$ & $34-77$ \\
Platinum sensitivity & & & \\
$\quad$ Sensitive & $9(69)$ & $0(0)$ & $4(31)$ \\
$\quad$ Resistant & $6(30)$ & $2(10)$ & $12(60)$ \\
$\quad$ Unknown & $3(60)$ & $1(20)$ & $1(20)$ \\
Cytoreduction status* & & & \\
$\quad$ Optimal & $10(50)$ & $1(5)$ & $9(45)$ \\
$\quad$ Suboptimal & $8(57)$ & $1(7)$ & $5(36)$ \\
$\quad$ No surgery & $0(0)$ & $1(50)$ & $1(50)$ \\
$\quad$ Unknown & $0(0)$ & $0(0)$ & $2(100)$ \\
\hline
\end{tabular}

Data are expressed as $n(\%)$ unless otherwise indicated.

*Cytoreduction status reported at the time of primary debulking surgery.

which germline CNAs substantially confound the LRR signal because of somatic CNA events across the TCGA data set and are thus candidates to be excluded from the PDX data. To rigorously define these outliers, a linear regression associating the total LRR value in the corrected data set to the total LRR value in the uncorrected one was fitted. The studentized residuals that resulted from the regression indicate by how many SDs genes diverge from the linear trend. A gene with residual magnitude $>4$ is expected to randomly occur with $P=6.3 \times 10^{-5}$ (the probability of a $\geq \pm 4 \sigma$ observation in a gaussian distribution, which appropriately models the residuals). In a data set of 23,803 genes, approximately 1.5 genes are expected to meet or exceed this criterion by chance alone. The regression residuals identified 224 genes exceeding this criterion (for a resulting false discovery rate of 1.5/ $224=0.0067$ across the entire genome). These are genes for which the somatic CNA signal is, with high confidence, substantially confounded by germline CNA events. These 224 genes were also removed from the xenograft data as genes whose signal is likely confounded by germline CNAs.

\section{Copy Number Status for Regions of Recurrent CNA Gain or Loss in Ovarian Carcinomas}

TCGA analysis defined regions of recurrent gain or loss in ovarian carcinomas. These regions and associated genes were downloaded from the TCGA supplemental data. ${ }^{13}$ The LRRs for genes in each region were extracted for each PDX, and then the mean LRR for each region was calculated. If the mean was positive, the maximum LRR observed for any gene in the region was taken as representative. When the mean LRR was negative, the minimum LRR for any gene in the region was used. These extreme values were considered representative because some recurrent CNA regions are defined fairly broadly, with the possibility that only a core 
A

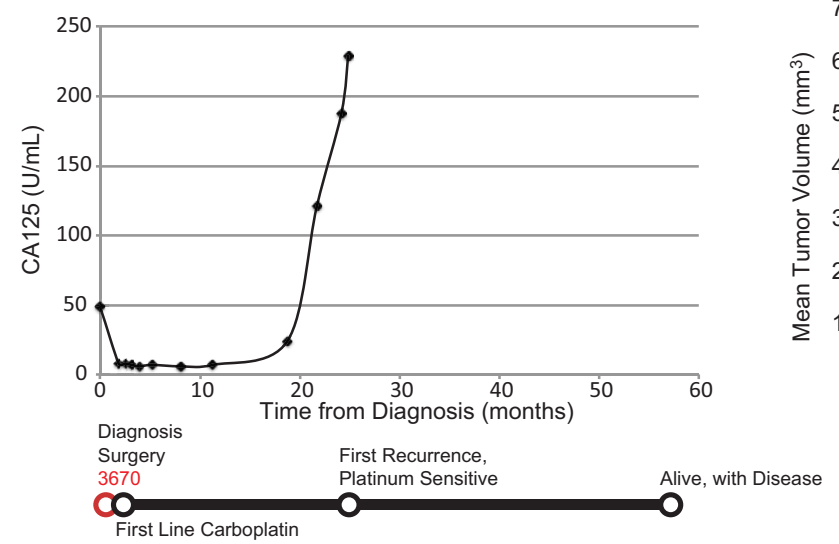

B

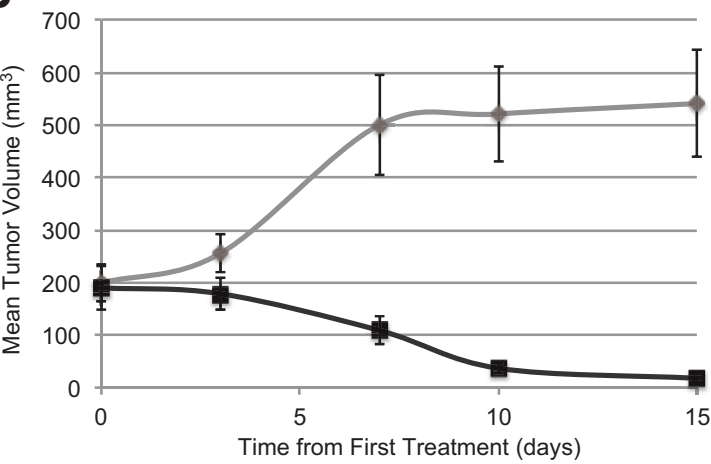

C

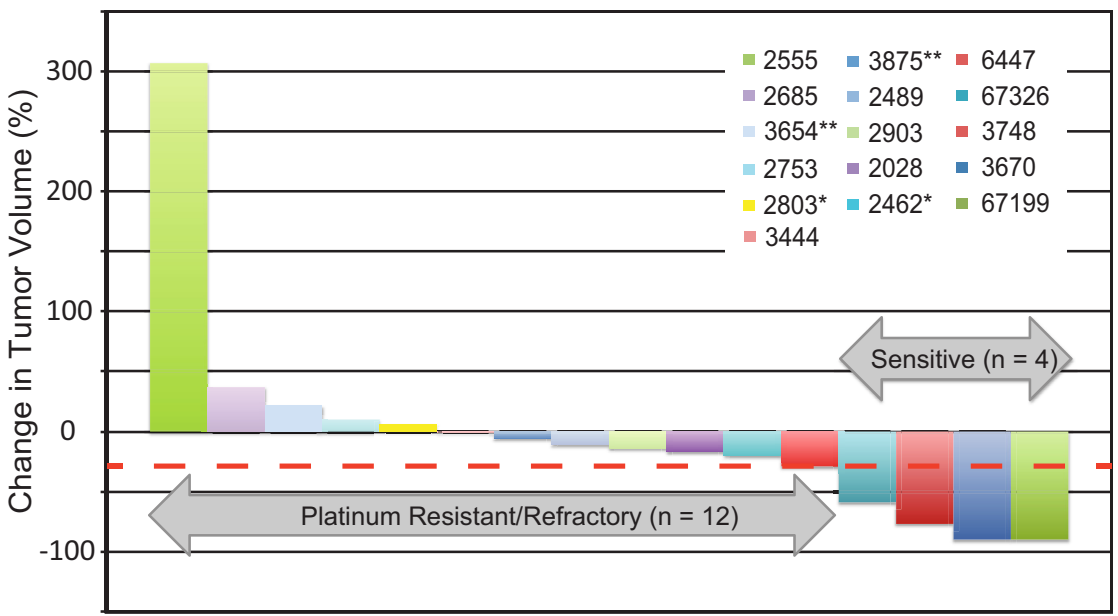

Xenograft Sensitivity to Carboplatin

Figure 1 Xenografts recapitulate patient response to carboplatin. A: Longitudinal CA125 values mapped onto the timeline of the disease course for case 3670. Clinical disease course is mapped below. Red circle indicates time at which the patient-derived xenograft (PDX) was established. B: Mean response of PDX model 3670 to carboplatin treatment. The gray line represents the untreated, control animals, whereas the black line represents the animals treated with two doses, 1 week apart, of carboplatin at $75 \mathrm{mg} / \mathrm{kg}$. Note that the response of this model to carboplatin corresponds to the platinum sensitivity of the parental tumor at the time of sampling (A). Additional examples are shown in Supplemental Figure S2. C: Summary of model responses to carboplatin. Sixteen models were treated with carboplatin as in B. PDXs from samples from the same patient acquired at different times are shown with asterisk and double asterisk. The dashed red line indicates the threshold for Response Evaluation Criteria for Solid Tumors criteria. Data are expressed as means \pm SEM (B). $n=10(\mathbf{B}) ; n=14$ (C).

of the region is highly amplified or homozygously lost and averaging the LRR values for all genes in the region would dilute this signal. An LRR $>0.3$ was considered amplified, an LRR $>0.15$ was considered a gain, a value $<-0.15$ was considered a heterozygous deletion, whereas an LRR $>-1$ was taken as a homozygous loss. These values were selected based on an examination of the LRR distributions for key ovarian oncogenes (CCNE1, MECOM, MYC) and tumor suppressors (PTEN, RB1, TP53).

\section{Gene Expression Analysis}

Small samples were shaved from snap-frozen PDX specimens for RNA extraction by the Trizol-based, Qiagen mRNA and RNA extraction kit (catalog number 217004,
Qiagen, Hilden, Germany). Sample quantity was assessed on a Nanodrop (ND-1000), and quality was evaluated with an Agilent bioanalyzer (Agilent, Santa Clara, CA). Gene expression analysis was performed on the Agilent platform $(8 \times 60,000$ array $)$. Data were preprocessed, normalized, and filtered using Robust Multichip Average normalization $^{27}$ to minimize batch variation and eliminate signals falling below the limit. Data were imported into GeneSpring GX version 7.3 (Agilent), which was used to conduct supervised and unsupervised hierarchical clustering. Additional statistical analyses were conducted in GeneSpring, using the algorithms provided in the package. Raw TCGA data were obtained from the International Cancer Genome Consortium (Rockville, MD) data portal (https://dcc.icgc. org). 
A

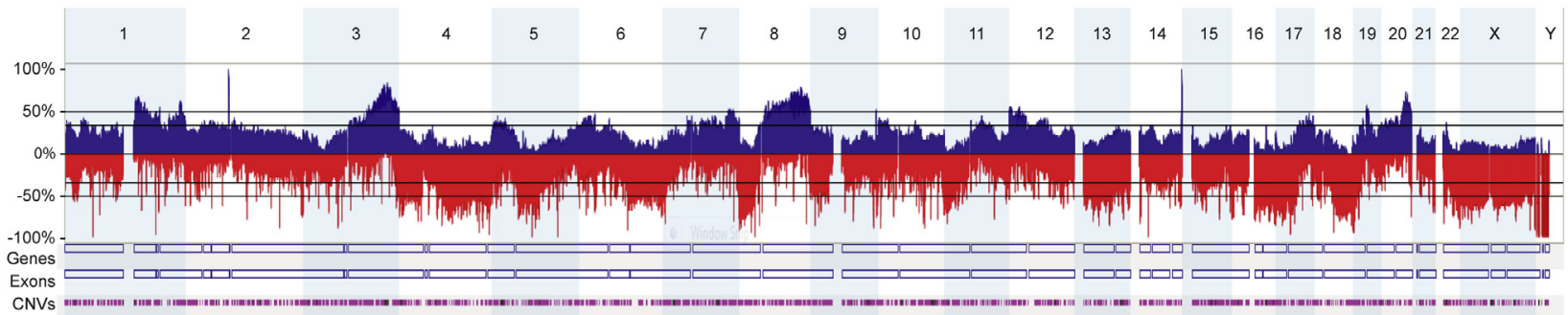

B

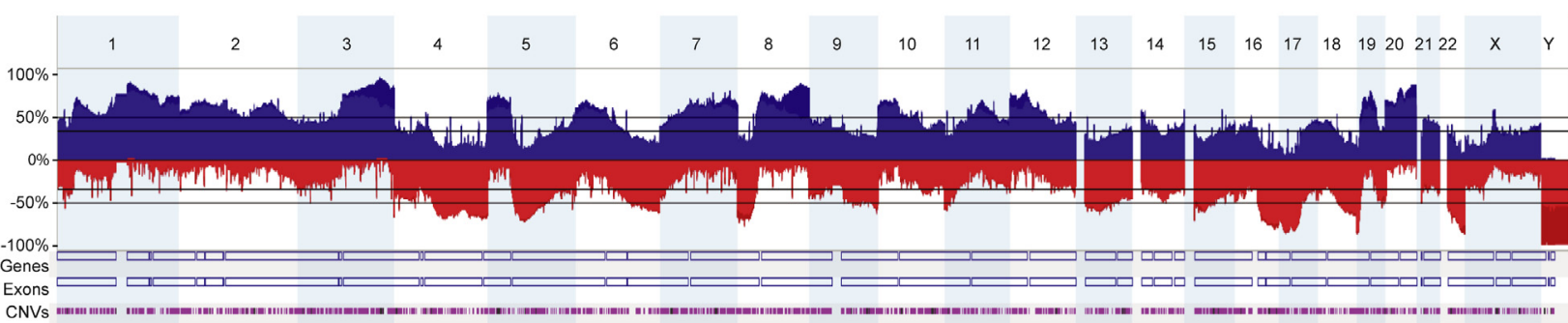

C

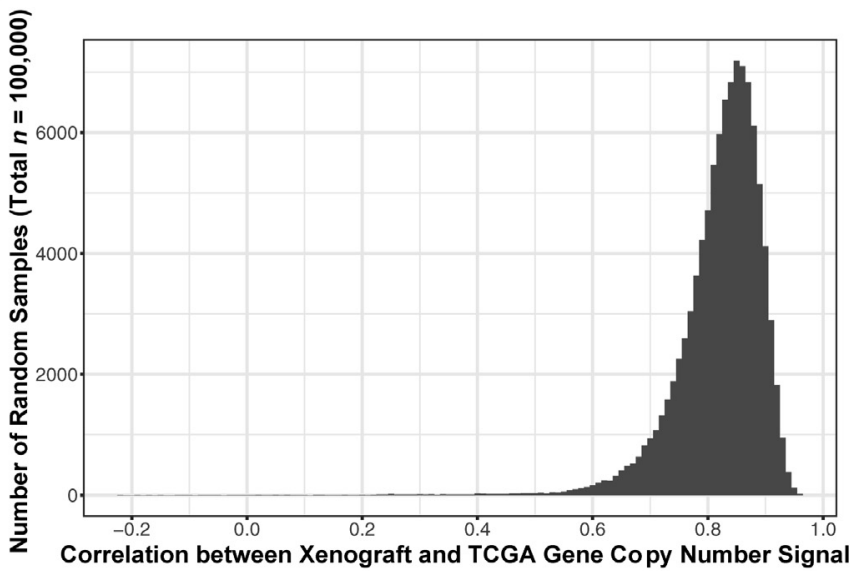

D

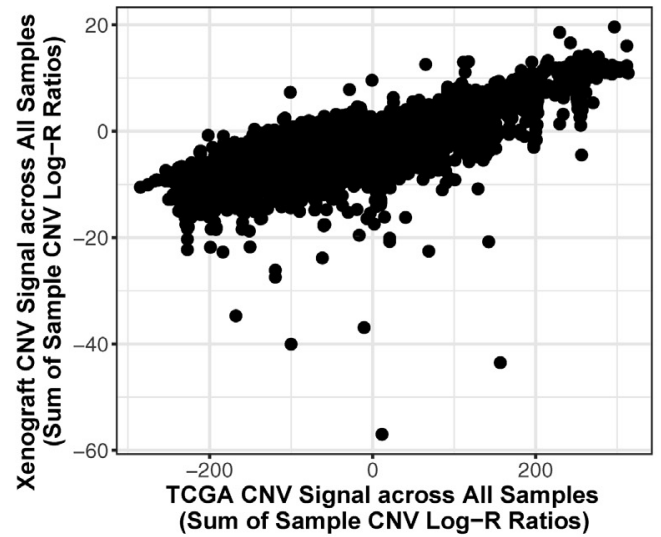

Figure 2 Copy number abnormalities (CNAs) in xenografts. A: All patient-derived xenografts (PDXs) were analyzed for CNAs. The overall proportion of cases with each CNA is shown in the top bar. Genes, exons, CNAs, and miRNAs are mapped below. B: All samples from The Cancer Genome Atlas (TCGA) were analyzed for CNAs using the variant-calling algorithms in A. C: All PDXs and the TCGA data were analyzed for CNAs. Correlation analysis was performed by randomly sampling one gene from each chromosome, calculating the correlation coefficient and repeating this 100,000 times. The median correlation was 0.84. D: CNAs were compared between TCGA data and PDXs. The CNA signal (array log-R ratio signal) for each gene, summed across the samples, was plotted for TCGA ( $x$ axis) and PDXs ( $y$ axis). Positive numbers indicate a propensity toward copy gains on average across samples, whereas negative numbers suggest a tendency toward copy loss. CNV, copy number variation.

Direct comparison to TCGA data required us to compile a list of overlapping probes and rewrite TCGA, PDX, and primary data files so that they only contained common probes. The commercial arrays used for our study contain 42,405 probes, whereas the TCGA analysis was conducted on two arrays, one containing 75,518 probes (version 2) and the final version of the array (version 3) containing 111,183 probes (Supplemental Figure S1). Preliminary analyses, as expected, demonstrated that batch normalization of the data were necessary, which was conducted using ComBAT, ${ }^{28}$ assuming both parametric and nonparametric data. All statistics were calculated using GeneSpring version GX7.3 (Agilent) and R Bioconductor (Bioconductor). ${ }^{29}$

\section{Results}

PDXs Recapitulate Patient Response to Platinum-Based Chemotherapy

PDXs were established by injecting fresh HGSC cell suspensions into the mammary fat pads of NOD/SCID or NSG mice. As reported previously, tumors can be generated at this site at high frequency ( $>90 \%$ take rate) and, on early passage of PDXs, reproduce the histology and immunophenotype of the primary tumor. ${ }^{17}$ Notably, some of the PDXs described in this study overlap with those described in our previous study, ${ }^{17}$ whereas others were 
A

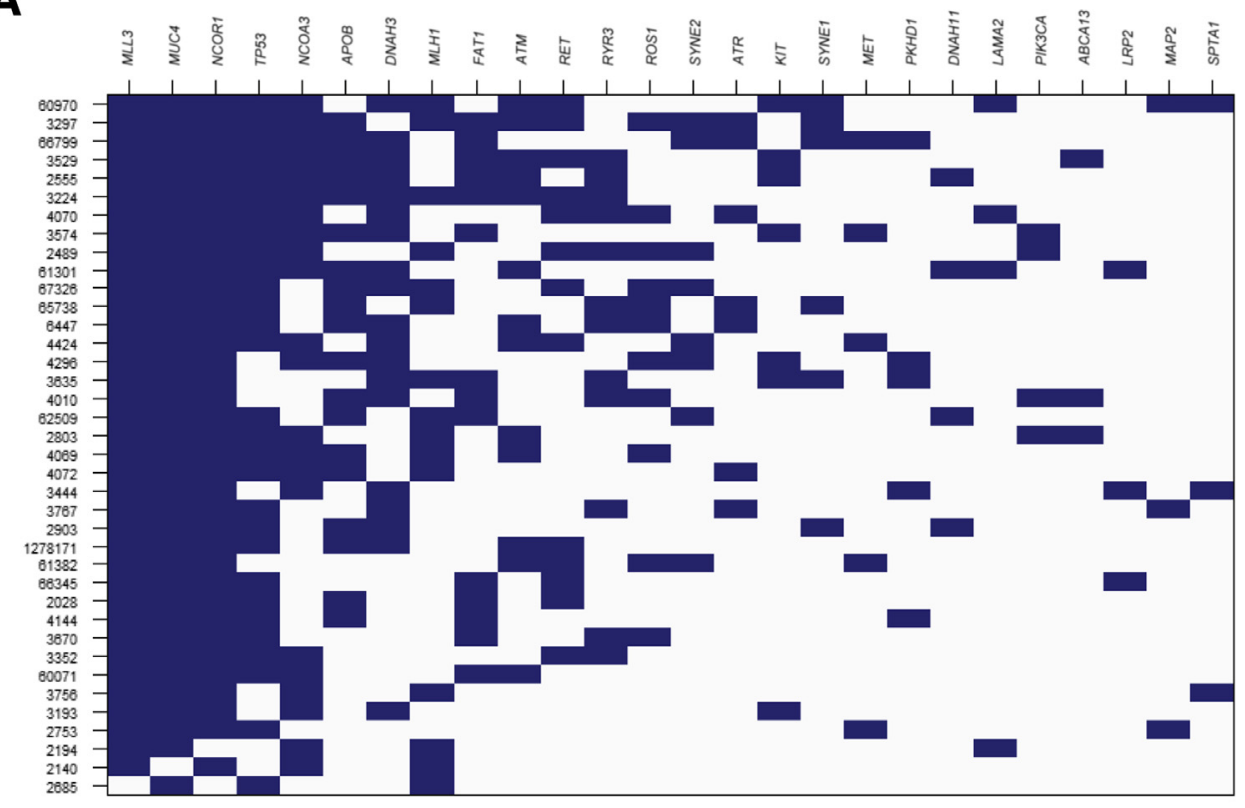

B

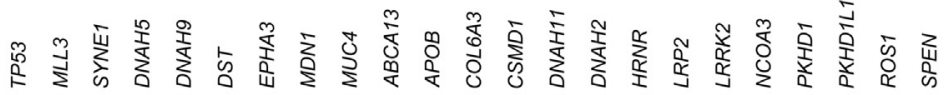

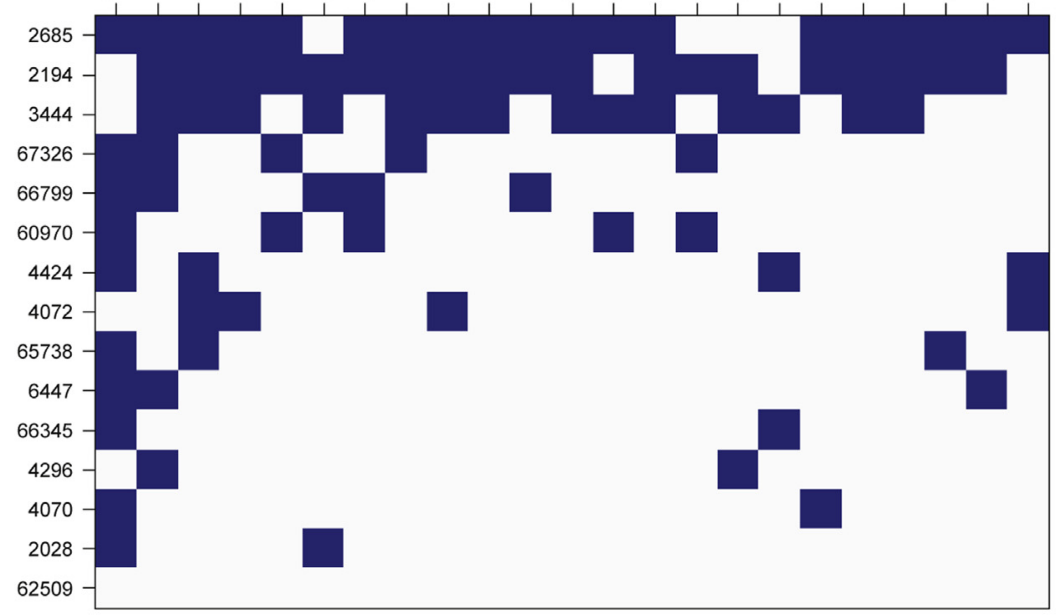

C

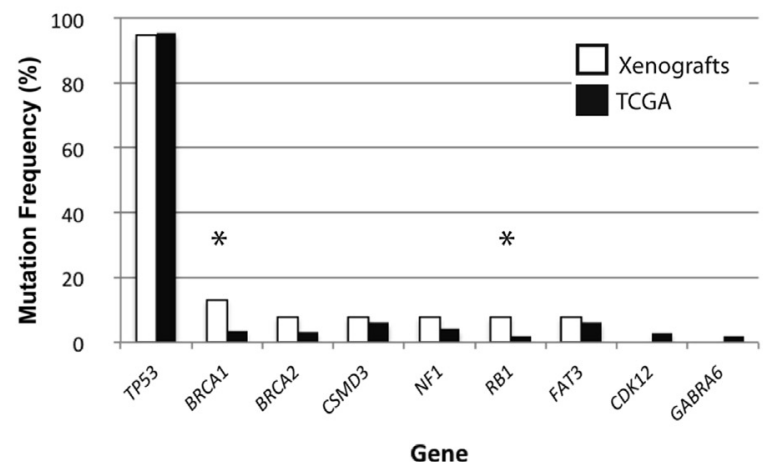

D

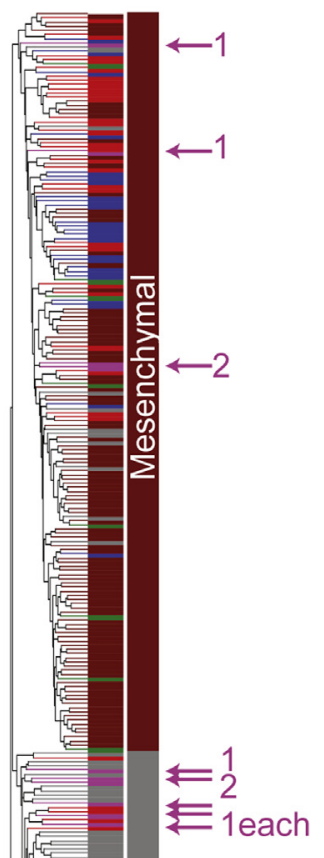

$\leftarrow 2$

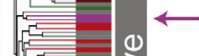

तั

은

$\leftarrow 2$

$\leftleftarrows 4$

$\leftarrow 8$

世

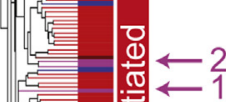

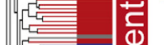

(ब)

青

$\leftarrow 3$ 
newly established after the time of publication. From our collection of PDXs, 38 were chosen for detailed analyses. These models were established from solid tumors or ascites at diagnosis or recurrence and represent the spectrum of disease observed in HGSC (Table 1). The complete clinical history and experimental details for each patient are listed in Supplemental Tables S1 and S3, respectively.

If PDXs are to serve as useful preclinical models, their response to standard-of-care chemotherapeutics should be similar to the patient's original tumor. The response to carboplatin of 16 PDX models from 14 patients was observed. Patients were monitored by longitudinal CA125 measurements, appropriate imaging, and clinic notes (Figure 1A and Supplemental Figure S1). PDX-bearing mice were treated with vehicle control or two murineequivalent doses of carboplatin $(75 \mathrm{mg} / \mathrm{kg})$, separated by 1 week. PDXs derived from patients whose tumors were adjudged clinically to be platinum sensitive at the time of sampling (Figure 1A) demonstrated a significant reduction in PDX size after two doses of carboplatin (Figure 1B). By contrast, PDXs from patients with tumors deemed platinum resistant at sample acquisition (Supplemental Figure S1, A and D) had a cytostatic response (Supplemental Figure S1, $\mathrm{B}$ and $\mathrm{C}$ ) or grew through treatment (Supplemental Figure S1E). All sample responses are shown in Supplemental Figure S2. Carboplatin responsiveness decreased with the emergence of platinum resistance in samples obtained from the same patient at different times in her clinical course (3654 versus 3875) (Supplemental Figure S1, B and C). Response Evaluation Criteria for Solid Tumors (RECIST), corresponding to $>30 \%$ reduction in tumor volume, was used to classify all PDXs tested as carboplatin sensitive or resistant (Figure 1C). By these criteria, platinum-sensitive PDXs corresponded to the patients who were platinum sensitive at the time of sampling. Two PDXs (67199 and 67326) were treated before the clinical responses of the cognate patients were known. For these prospective cases, the PDX response accurately predicted that of the patient (Figure 1C). Similarly, the platinum-resistant (by RECIST) PDXs were derived from patients who demonstrated platinum resistance at the time of sampling.

\section{PDXs Display Genetic Alterations Representative of HGSC}

Copy number abnormalities (CNAs) were analyzed to assess how well PDXs represent the genomic diversity of HGSC (Figure 2A). Comparison to the CNA analysis in the TCGA data set (Figure 2B) demonstrated a similar overall pattern of alteration across the PDX collection. One gene per chromosome was randomly sampled, the correlation coefficient between PDX and TCGA primary tumor data across the genome was calculated, and this process was repeated 100,000 times to evaluate this similarity statistically (Figure 2C). This analysis generated a median correlation coefficient of 0.84 , consistent with the close association observed (Figure 2D) between total xenograft and TCGA sample copy number signals.

To assess the mutational landscape of the PDXs, targeted sequencing was performed using a custom panel based on the most frequently mutated genes in ovarian cancer (Supplemental Table S4) to a mean target coverage of $628 \times$ (Figure 3A). Of the 38 samples, 15 had matched normal DNA available to distinguish somatic from germline alterations (Figure 3B). Overall, 93\% of the PDXs demonstrated genetic alterations of TP53, consistent with the TCGA data, with only three lacking a TP53 abnormality. Manual review of the TP53 locus in mutated and nonmutated samples showed additional deletions, splicing variants, and frameshift mutations (Supplemental Table S2). Specifically, one of the other three cases had very low TP53 mRNA levels (2194), potentially reflecting epigenetic silencing; whereas the other two $(4010,61382)$ had high $M D M 2$ expression, which might result in TP53 degradation. BRCA1 and $B R C A 2$ were mutated at frequencies of $13 \%$ and $8 \%$, respectively (Figure 3C). All six cases with clinically reported germline BRCA1/2 mutations (PDX cases 3444, 3574, 4070, 61301, 66799, and 1278171) were confirmed by sequencing the PDX and/or normal DNA. Comparison with TCGA study ${ }^{13}$ showed that the mutational spectrum of our PDXs and primary HGSCs was similar (Figure 3C), with the exception of $R B I$ and BRCAl, which showed a higher frequency of mutations in PDXs than TCGA (Supplemental Table S1). Notably, however, the published TCGA data from cBioPortal (Center for Molecular

Figure 3 Xenografts represent the genetic diversity of high-grade serous ovarian cancer (HGSC). A: Targeted sequencing of the 115 most highly mutated genes in breast and ovarian cancer was conducted for all patient-derived xenografts (PDXs), and mutations (somatic and germline, where possible to assess) were called. Genes are arranged from most to least mutated, with mutated genes shown in blue and nonmutated genes for each PDX shown in white. Some genes are large and tend to carry single-nucleotide polymorphisms (SNPs) that are not filtered out by the SNP database, resulting in apparently higher mutation rates than might be expected. These genes include MLL3, MUC4, and NCOR1. Where matched normal DNA was available, it was possible to call somatic variants (removing the effects of these germline SNPs), as shown in C. B: Fifteen cases had matched normal blood available, which was also sequenced to make somatic variant calls. Genes are arranged from most to least mutated, and only genes with somatic variants are shown. Mutations are indicated by blue boxes. Reported pathogenic germline mutations in BRCA1 and BRCA2 were confirmed (Supplemental Table S1). C: The most highly mutated genes in The Cancer Genome Atlas (TCGA) were mutated at similar frequencies in the PDXs. Comparisons with the frequencies of mutation observed in the TCGA were calculated using a $\chi^{2}$ statistic, which was significantly different for BRCA1 and RB1 but not the other genes assessed. D: Clustering of TCGA samples and our PDX collection, based on the genes in TCGA publication most differentially expressed among the four subgroups of HGSC. Note that PDXs scatter throughout the gene expression-defined subgroups, except the immunoreactive group. The number of samples represented by each arrow is indicated to the right. ${ }^{*} P<0.05$ versus TCGA data. 
Oncology, Memorial Sloan Kettering Cancer Center, New York, NY; http://www.cbioportal.org) was also retrieved, and it was found that the rate of mutations seen in BRCAl (11\%) was much higher than in the initial TCGA report and much closer to that seen in the PDXs. Hence, it could well be that only $R B 1$ is mutated at a higher frequency in PDXs compared with TCGA.

\section{PDXs Recapitulate Ovarian Cancer Heterogeneity}

The mRNA expression profiles of the PDXs were compared with TCGA data ${ }^{13}$ rather than the Tothill data because the former used Agilent arrays (as did we), whereas the latter used the Affymetrix platform. The TCGA used a customized microarray, which is not commercially available. To facilitate this analysis, a list of TCGA probes that overlapped with our arrays was generated. Conversion of all TCGA and PDX data sets into new data files that contain only common probes enabled comparison of these data sets.

Clustering was performed based on the TCGA subtype signature that was most useful for stratifying HGSC into the four subgroups (Figure 3D). As expected, when clustered by these signatures, the TCGA samples clustered into the four subgroups seen in their initial report. By contrast, PDXs were stratified into three subtypes, with the immunoreactive group missing, as expected (given the use of NSG mice). In addition, only 4 of 38 PDXs were stratified into the mesenchymal subgroup. Notably, the analysis assigned some TCGA samples to other subtypes than originally reported. ${ }^{13}$ This discrepancy could reflect differences in clustering algorithms, imperfection in subtype definitions based on this gene signature as reported previously, ${ }^{14}$ and/or reduction in the number of probes available for data comparison. For convenient reference, the genomic features of each PDX are aggregated in a printable summary sheet (Supplemental Table S5) and are available on a searchable, interactive website (https:// xenografts.shinyapps.io/summarysheet, last accessed March 6, 2018).

\section{Discussion}

PDXs have been proposed to be more clinically relevant models than available cancer cell lines and genetically engineered mouse models. However, with the exception of reports on colorectal cancer PDXs, ${ }^{30,31}$ only a few small studies have characterized PDXs of any tumor type for their response to chemotherapy, phenotype, and/or genotype ${ }^{32-34}$ A substantial number of HGSC PDXs annotated for CNA, transcriptional subgroup, and mutational analysis were developed. These PDXs have CNAs and mutational spectra that represent interpatient diversity and accurately recapitulate the (matched) patient response to platinum chemotherapy. This genomically characterized PDX collection may provide an improved preclinical model of HGSC with potential utility in drug discovery.
Ovarian cancer PDXs have been described previously, with variable degrees of characterization of their response to standard-of-care chemotherapy, ${ }^{34-36}$ immunohistochemical features, ${ }^{37-39}$ and specific genetic alterations. ${ }^{8,34,40}$ These studies used a small number of serous cancer samples ( 3 to 16 per study), and their general applicability remained unclear. The largest, most comprehensive study examined gene expression, exon-specific mutations in seven genes, and copy number of eight genes in PDXs derived from 16 serous cancers and 18 epithelial ovarian cancers of other histotypes. ${ }^{41}$ In that report, Ricci et $\mathrm{al}^{41}$ demonstrated that PDXs in the subcutaneous or intraperitoneal sites recapitulate the gross and histologic appearance, as well as at least one mutation of their matched human ovarian cancer. They identified more CNAs in their PDX cohort, compared with the matched primary tumor, based on the eight genes examined and found differences in gene expression between PDXs and matched primary tumors $(n=9)$, largely reflecting immune response genes. Finally, they examined PDX response to cisplatin and paclitaxel, with 6 of 11 showing similar sensitivity as the primary tumor. Notably, these authors reported a tumor take rate of only $25 \%$, in contrast to our $>90 \%$ take rate, likely reflecting the use of nude instead of NSG mice and/or the use of a less sensitive engraftment site. ${ }^{17}$ The low take rate could bias the pool of PDXs available for analysis and the conclusions of this study. Furthermore, en masse analysis of all epithelial ovarian cancer histotypes could complicate the interpretation of these results. For example, of the 6 of 11 PDXs that showed a response to platinum-based chemotherapy that correlated with the primary tumor response, 4 of 5 were HGSC, making their data more consistent with ours. Regardless, the current study provides the largest and most indepth molecular characterization of clinically annotated HGSC PDX reported to date.

Various reports have suggested that PDXs might be useful for directing personalized medicine approaches. ${ }^{42-44} \mathrm{Hi}$ dalgo et $\mathrm{al}^{42}$ found that for various solid tumors, not including HGSC, PDX models could be used to test and select treatment options, resulting in durable partial remissions in 15 of 17 treatments administered. Others have suggested good correlation among immunohistochemical, ${ }^{20,45-47}$ gene expression, ${ }^{20,31,45-50}$ copy number alteration, ${ }^{30,31,45,46,50}$ and selected gene mutations between solid tumors ${ }^{48,49,51}$ and their matched PDXs in colorectal, ${ }^{30,31}$ breast, ${ }^{45-47,52}$ lung, ${ }^{20}$ pancreatic, ${ }^{48,51}$ head and neck, ${ }^{49}$ and renal cancers. ${ }^{50}$ The results show that HGSC PDXs also effectively recapitulate the spectrum of genetic alterations in this disease. The frequency of manually vetted alterations in TP53 was as high as expected. ${ }^{13}$ In addition, all clinically reported mutations in BRCA1/2 in the PDX or germline data were validated (where available). Furthermore, the PDXs recapitulate the clinical response of patient tumors to carboplatin. These findings are consistent with reports on other tumor types ${ }^{30,52}$ and smaller studies of various ovarian cancer subtypes. ${ }^{20,32}$ The recapitulation of response to standard of care in conjunction with the diversity in genetic alterations and gene expression patterns of 
PDX suggest that they have the potential to be used as representative preclinical models of HGSC.

The use of PDXs in preclinical studies continues to increase, along with the promise that these models will increase the success of investigational therapeutics. Credentialing PDX models of HGSC should speed progress in HGSC research by providing improved preclinical models of standard of care that can be used to test novel targets and more accurately evaluate their likelihood of success. Nevertheless, PDX models have intrinsic limitations, most prominently, their inability to model the antitumor immune response, a deficiency writ large by the recent success of antitumor immune therapy. ${ }^{53}$ Although better humanized mice have been reported recently, it will be difficult, if not impossible, to replace all the stromal cytokines, chemokines, and growth factors that differ in mice and humans. ${ }^{54}$ Finally, the practicality of PDXs, including the cost of their establishment and maintenance, also could limit wide applicability.

\section{Acknowledgments}

We thank the patients, surgeons (Drs. Joan Murphy, Barry Rosen, Jason Dodge, Sarah Ferguson, and Stephane Laframboise), and tissue bank staff who contributed the biospecimens to this project; and the Princess Margaret Genomics Centre.

\section{Supplemental Data}

Supplemental material for this article can be found at https://doi.org/10.1016/j.ajpath.2018.01.019.

\section{References}

1. Siegel RL, Miller KD, Jemal A: Cancer statistics, 2017. CA Cancer J Clin 2017, 67:7-30

2. Cannistra SA: Cancer of the ovary. N Engl J Med 2004, 351: 2519-2529

3. McGuire WP, Hoskins WJ, Brady MF, Kucera PR, Partridge EE, Look KY, Clarke-Pearson DL, Davidson M: Cyclophosphamide and cisplatin compared with paclitaxel and cisplatin in patients with stage III and stage IV ovarian cancer. N Engl J Med 1996, 334:1-6

4. Ozols RF, Bundy BN, Greer BE, Fowler JM, Clarke-Pearson D, Burger RA, Mannel RS, DeGeest K, Hartenbach EM, Baergen R: Phase III trial of carboplatin and paclitaxel compared with cisplatin and paclitaxel in patients with optimally resected stage III ovarian cancer: a Gynecologic Oncology Group study. J Clin Oncol 2003, 21 : 3194-3200

5. du Bois A, Luck HJ, Meier W, Adams HP, Mobus V, Costa S, Bauknecht T, Richter B, Warm M, Schroder W, Olbricht S, Nitz U, Jackisch C, Emons G, Wagner U, Kuhn W, Pfisterer J: A randomized clinical trial of cisplatin/paclitaxel versus carboplatin/paclitaxel as first-line treatment of ovarian cancer. J Natl Cancer Inst 2003, 95: $1320-1329$

6. Siegel R, Ma J, Zou Z, Jemal A: Cancer statistics, 2014. CA Cancer J Clin 2014, 64:9-29

7. Jandial DD, Messer K, Farshchi-Heydari S, Pu M, Howell SB: Tumor platinum concentration following intraperitoneal administration of cisplatin versus carboplatin in an ovarian cancer model. Gynecol Oncol 2009, 115:362-366

8. Press JZ, Kenyon JA, Xue H, Miller MA, De Luca A, Miller DM, Huntsman DG, Gilks CB, McAlpine JN, Wang YZ: Xenografts of primary human gynecological tumors grown under the renal capsule of NOD/SCID mice show genetic stability during serial transplantation and respond to cytotoxic chemotherapy. Gynecol Oncol 2008, 110:256-264

9. Markman M: Optimal management of recurrent ovarian cancer. Int J Gynecol Cancer 2009, 19 Suppl 2:S40-S43

10. Pujade-Lauraine E, Hilpert F, Weber B, Reuss A, Poveda A, Kristensen G, Sorio R, Vergote I, Witteveen P, Bamias A, Pereira D, Wimberger P, Oaknin A, Mirza MR, Follana P, Bollag D, Ray-Coquard I: Bevacizumab combined with chemotherapy for platinum-resistant recurrent ovarian cancer: the AURELIA open-label randomized phase III trial. J Clin Oncol 2014, 32: 1302-1308

11. Mirza MR, Monk BJ, Herrstedt J, Oza AM, Mahner S, Redondo A, Fabbro M, Ledermann JA, Lorusso D, Vergote I, Ben-Baruch NE, Marth C, Madry R, Christensen RD, Berek JS, Dorum A, Tinker AV, du Bois A, Gonzalez-Martin A, Follana P, Benigno B, Rosenberg P, Gilbert L, Rimel BJ, Buscema J, Balser JP, Agarwal S, Matulonis UA; ENGOT-OV16/NOVA Investigators: Niraparib maintenance therapy in platinum-sensitive, recurrent ovarian cancer. N Engl J Med 2016, 375:2154-2164

12. Tothill RW, Tinker AV, George J, Brown R, Fox SB, Lade S, Johnson DS, Trivett MK, Etemadmoghadam D, Locandro B, Traficante N, Fereday S, Hung JA, Chiew YE, Haviv I, Gertig D, DeFazio A, Bowtell DD: Novel molecular subtypes of serous and endometrioid ovarian cancer linked to clinical outcome. Clin Cancer Res 2008, 14:5198-5208

13. Cancer Genome Atlas Research Network: Integrated genomic analyses of ovarian carcinoma. Nature 2011, 474:609-615

14. Verhaak RG, Tamayo P, Yang JY, Hubbard D, Zhang H, Creighton CJ, et al; Cancer Genome Atlas Research Network: Prognostically relevant gene signatures of high-grade serous ovarian carcinoma. J Clin Invest 2013, 123:517-525

15. Domcke S, Sinha R, Levine DA, Sander C, Schultz N: Evaluating cell lines as tumour models by comparison of genomic profiles. Nat Commun 2013, 4:2126

16. Shaw TJ, Senterman MK, Dawson K, Crane CA, Vanderhyden BC: Characterization of intraperitoneal, orthotopic, and metastatic xenograft models of human ovarian cancer. Mol Ther 2004, 10: $1032-1042$

17. Stewart JM, Shaw PA, Gedye C, Bernardini MQ, Neel BG, Ailles LE: Phenotypic heterogeneity and instability of human ovarian tumor-initiating cells. Proc Natl Acad Sci U S A 2011, 108: $6468-6473$

18. Reagan-Shaw S, Nihal M, Ahmad N: Dose translation from animal to human studies revisited. FASEB J 2008, 22:659-661

19. Merk J, Rolff J, Becker M, Leschber G, Fichtner I: Patient-derived xenografts of non-small-cell lung cancer: a pre-clinical model to evaluate adjuvant chemotherapy? Eur J Cardiothorac Surg 2009, 36: 454-459

20. Fichtner I, Rolff J, Soong R, Hoffmann J, Hammer S, Sommer A, Becker M, Merk J: Establishment of patient-derived non-small cell lung cancer xenografts as models for the identification of predictive biomarkers. Clin Cancer Res 2008, 14: 6456-6468

21. Das B, Antoon R, Tsuchida R, Lotfi S, Morozova O, Farhat W, Malkin D, Koren G, Yeger H, Baruchel S: Squalene selectively protects mouse bone marrow progenitors against cisplatin and carboplatin-induced cytotoxicity in vivo without protecting tumor growth. Neoplasia 2008, 10:1105-1119

22. Conway T, Wazny J, Bromage A, Tymms M, Sooraj D, Williams ED, Beresford-Smith B: Xenome-a tool for classifying reads from xenograft samples. Bioinformatics 2012, 28:i172-i178 
23. Li H, Durbin R: Fast and accurate long-read alignment with BurrowsWheeler transform. Bioinformatics 2010, 26:589-595

24. Wang K, Li M, Hakonarson H: ANNOVAR: functional annotation of genetic variants from next-generation sequencing data. Nucleic Acids Res 2010, 38:e164

25. Karolchik D, Hinrichs AS, Furey TS, Roskin KM, Sugnet CW, Haussler D, Kent WJ: The UCSC Table Browser data retrieval tool. Nucleic Acids Res 2004, 32:D493-D496

26. Kent WJ, Sugnet CW, Furey TS, Roskin KM, Pringle TH, Zahler AM, Haussler D: The human genome browser at UCSC Genome Res 2002, 12:996-1006

27. Irizarry RA, Hobbs B, Collin F, Beazer-Barclay YD, Antonellis KJ, Scherf U, Speed TP: Exploration, normalization, and summaries of high density oligonucleotide array probe level data. Biostatistics 2003, 4:249-264

28. Johnson WE, Li C, Rabinovic A: Adjusting batch effects in microarray expression data using empirical Bayes methods. Biostatistics 2007, 8:118-127

29. Gentleman RC, Carey VJ, Bates DM, Bolstad B, Dettling M, Dudoit S, Ellis B, Gautier L, Ge Y, Gentry J, Hornik K, Hothorn $\mathrm{T}$, Huber $\mathrm{W}$, Iacus $\mathrm{S}$, Irizarry $\mathrm{R}$, Leisch $\mathrm{F}$, Li C, Maechler M, Rossini AJ, Sawitzki G, Smith C, Smyth G, Tierney L, Yang JY, Zhang J: Bioconductor: open software development for computational biology and bioinformatics. Genome Biol 2004, 5:R80

30. Bertotti A, Migliardi G, Galimi F, Sassi F, Torti D, Isella C, Cora D, Di Nicolantonio F, Buscarino M, Petti C, Ribero D, Russolillo N, Muratore A, Massucco P, Pisacane A, Molinaro L, Valtorta E, Sartore-Bianchi A, Risio M, Capussotti L, Gambacorta M, Siena S, Medico E, Sapino A, Marsoni S, Comoglio PM, Bardelli A, Trusolino L: A molecularly annotated platform of patient-derived xenografts ("xenopatients") identifies HER2 as an effective therapeutic target in cetuximab-resistant colorectal cancer. Cancer Discov 2011, 1:508-523

31. Julien S, Merino-Trigo A, Lacroix L, Pocard M, Goere D, Mariani P, Landron S, Bigot L, Nemati F, Dartigues $P$, Weiswald LB, Lantuas D, Morgand L, Pham E, Gonin P, Dangles-Marie V, Job B, Dessen P, Bruno A, Pierre A, De The H, Soliman H, Nunes M, Lardier G, Calvet L, Demers B, Prevost G, Vrignaud P, RomanRoman S, Duchamp O, Berthet C: Characterization of a large panel of patient-derived tumor xenografts representing the clinical heterogeneity of human colorectal cancer. Clin Cancer Res 2012, 18: 5314-5328

32. Liu JF, Palakurthi S, Zeng Q, Zhou S, Ivanova E, Huang W, Zervantonakis IK, Selfors LM, Shen Y, Pritchard CC, Zheng M, Adleff V, Papp E, Piao H, Novak M, Fotheringham S, Wulf GM, English J, Kirschmeier PT, Velculescu VE, Paweletz C, Mills GB, Livingston DM, Brugge JS, Matulonis UA, Drapkin R: Establishment of patient-derived tumor xenograft models of epithelial ovarian cancer for preclinical evaluation of novel therapeutics. Clin Cancer Res 2017, 23:1263-1273

33. Scott CL, Becker MA, Haluska P, Samimi G: Patient-derived xenograft models to improve targeted therapy in epithelial ovarian cancer treatment. Front Oncol 2013, 3:295

34. Weroha SJ, Becker MA, Enderica-Gonzalez S, Harrington SC, Oberg AL, Maurer MJ, Perkins SE, AlHilli M, Butler KA, McKinstry S, Fink S, Jenkins RB, Hou X, Kalli KR, Goodman KM, Sarkaria JN, Karlan BY, Kumar A, Kaufmann SH, Hartmann LC, Haluska P: Tumorgrafts as in vivo surrogates for women with ovarian cancer. Clin Cancer Res 2014, 20:1288-1297

35. Topp MD, Hartley L, Cook M, Heong V, Boehm E, McShane L, Pyman J, McNally O, Ananda S, Harrell M, Etemadmoghadam D, Galletta L, Alsop K, Mitchell G, Fox SB, Kerr JB, Hutt KJ, Kaufmann SH; Australian Ovarian Cancer Study, Swisher EM, Bowtell DD, Wakefield MJ, Scott CL: Molecular correlates of platinum response in human high-grade serous ovarian cancer patientderived xenografts. Mol Oncol 2014, 8:656-668
36. George E, Kim H, Krepler C, Wenz B, Makvandi M, Tanyi JL, Brown E, Zhang R, Brafford P, Jean S, Mach RH, Lu Y, Mills GB, Herlyn M, Morgan M, Zhang X, Soslow R, Drapkin R, Johnson N, Zheng Y, Cotsarelis G, Nathanson KL, Simpkins F: A patientderived-xenograft platform to study BRCA-deficient ovarian cancers. JCI Insight 2017, 2:e89760

37. Lee CH, Xue H, Sutcliffe M, Gout PW, Huntsman DG, Miller DM, Gilks CB, Wang YZ: Establishment of subrenal capsule xenografts of primary human ovarian tumors in SCID mice: potential models. Gynecol Oncol 2005, 96:48-55

38. Bankert RB, Balu-Iyer SV, Odunsi K, Shultz LD, Kelleher RJ Jr, Barnas JL, Simpson-Abelson M, Parsons R, Yokota SJ: Humanized mouse model of ovarian cancer recapitulates patient solid tumor progression, ascites formation, and metastasis. PLoS One 2011, 6: e24420

39. Dong R, Qiang W, Guo H, Xu X, Kim JJ, Mazar A, Kong B, Wei JJ: Histologic and molecular analysis of patient derived xenografts of high-grade serous ovarian carcinoma. J Hematol Oncol 2016, 9:92

40. AlHilli MM, Becker MA, Weroha SJ, Flatten KS, Hurley RM, Harrell MI, Oberg AL, Maurer MJ, Hawthorne KM, Hou X, Harrington SC, McKinstry S, Meng XW, Wilcoxen KM, Kalli KR, Swisher EM, Kaufmann SH, Haluska P: In vivo anti-tumor activity of the PARP inhibitor niraparib in homologous recombination deficient and proficient ovarian carcinoma. Gynecol Oncol 2016, 143: 379-388

41. Ricci F, Bizzaro F, Cesca M, Guffanti F, Ganzinelli M, Decio A, Ghilardi C, Perego P, Fruscio R, Buda A, Milani R, Ostano P, Chiorino G, Bani MR, Damia G, Giavazzi R: Patient-derived ovarian tumor xenografts recapitulate human clinicopathology and genetic alterations. Cancer Res 2014, 74:6980-6990

42. Hidalgo M, Bruckheimer E, Rajeshkumar NV, Garrido-Laguna I, De Oliveira E, Rubio-Viqueira B, Strawn S, Wick MJ, Martell J, Sidransky D: A pilot clinical study of treatment guided by personalized tumorgrafts in patients with advanced cancer. Mol Cancer Ther 2011, 10:1311-1316

43. Lee WS, Kim HY, Seok JY, Jang HH, Park YH, Kim SY, Shin DB, Hong S: Genomic profiling of patient-derived colon cancer xenograft models. Medicine (Baltimore) 2014, 93:e298

44. Stebbing J, Paz K, Schwartz GK, Wexler LH, Maki R, Pollock RE, Morris R, Cohen R, Shankar A, Blackman G, Harding V, Vasquez D, Krell J, Ciznadija D, Katz A, Sidransky D: Patient-derived xenografts for individualized care in advanced sarcoma. Cancer 2014, 120: 2006-2015

45. Reyal F, Guyader C, Decraene C, Lucchesi C, Auger N, Assayag F, De Plater L, Gentien D, Poupon MF, Cottu P, De Cremoux P, Gestraud P, Vincent-Salomon A, Fontaine JJ, Roman-Roman S, Delattre O, Decaudin D, Marangoni E: Molecular profiling of patientderived breast cancer xenografts. Breast Cancer Res 2012, 14:R11

46. Marangoni E, Vincent-Salomon A, Auger N, Degeorges A, Assayag F, de Cremoux P, de Plater L, Guyader C, De Pinieux G, Judde JG, Rebucci M, Tran-Perennou C, Sastre-Garau X, SigalZafrani B, Delattre O, Dieras V, Poupon MF: A new model of patient tumor-derived breast cancer xenografts for preclinical assays. Clin Cancer Res 2007, 13:3989-3998

47. DeRose YS, Wang G, Lin YC, Bernard PS, Buys SS, Ebbert MT, Factor R, Matsen C, Milash BA, Nelson E, Neumayer L, Randall RL, Stijleman IJ, Welm BE, Welm AL: Tumor grafts derived from women with breast cancer authentically reflect tumor pathology, growth, metastasis and disease outcomes. Nat Med 2011, 17: $1514-1520$

48. Garrido-Laguna I, Uson M, Rajeshkumar NV, Tan AC, de Oliveira E, Karikari C, Villaroel MC, Salomon A, Taylor G, Sharma R, Hruban RH, Maitra A, Laheru D, Rubio-Viqueira B, Jimeno A, Hidalgo M: Tumor engraftment in nude mice and enrichment in stroma- related gene pathways predict poor survival and resistance to gemcitabine in patients with pancreatic cancer. Clin Cancer Res 2011, $17: 5793-5800$ 
49. Keysar SB, Astling DP, Anderson RT, Vogler BW, Bowles DW, Morton JJ, Paylor JJ, Glogowska MJ, Le PN, Eagles-Soukup JR, Kako SL, Takimoto SM, Sehrt DB, Umpierrez A, Pittman MA, Macfadden SM, Helber RM, Peterson S, Hausman DF, Said S, Leem TH, Goddard JA, Arcaroli JJ, Messersmith WA, Robinson WA, Hirsch FR, Varella-Garcia M, Raben D, Wang XJ, Song JI, Tan AC, Jimeno A: A patient tumor transplant model of squamous cell cancer identifies PI3K inhibitors as candidate therapeutics in defined molecular bins. Mol Oncol 2013, 7:776-790

50. Sivanand S, Pena-Llopis S, Zhao H, Kucejova B, Spence P, PaviaJimenez A, Yamasaki T, McBride DJ, Gillen J, Wolff NC, Morlock L, Lotan Y, Raj GV, Sagalowsky A, Margulis V, Cadeddu JA, Ross MT, Bentley DR, Kabbani W, Xie XJ, Kapur P, Williams NS, Brugarolas J: A validated tumorgraft model reveals activity of dovitinib against renal cell carcinoma. Sci Transl Med 2012, 4:137ra75

51. Rubio-Viqueira B, Jimeno A, Cusatis G, Zhang X, IacobuzioDonahue C, Karikari C, Shi C, Danenberg K, Danenberg PV,
Kuramochi H, Tanaka K, Singh S, Salimi-Moosavi H, Bouraoud N, Amador ML, Altiok S, Kulesza P, Yeo C, Messersmith W, Eshleman J, Hruban RH, Maitra A, Hidalgo M: An in vivo platform for translational drug development in pancreatic cancer. Clin Cancer Res 2006, 12:4652-4661

52. Zhang X, Claerhout S, Prat A, Dobrolecki LE, Petrovic I, Lai Q, Landis MD, Wiechmann L, Schiff R, Giuliano M, Wong H, Fuqua SW, Contreras A, Gutierrez C, Huang J, Mao S, Pavlick AC, Froehlich AM, Wu MF, Tsimelzon A, Hilsenbeck SG, Chen ES, Zuloaga P, Shaw CA, Rimawi MF, Perou CM, Mills GB, Chang JC, Lewis MT: A renewable tissue resource of phenotypically stable, biologically and ethnically diverse, patient-derived human breast cancer xenograft models. Cancer Res 2013, 73:4885-4897

53. Restifo NP, Smyth MJ, Snyder A: Acquired resistance to immunotherapy and future challenges. Nat Rev Cancer 2016, 16:121-126

54. Shultz LD, Brehm MA, Garcia-Martinez JV, Greiner DL: Humanized mice for immune system investigation: progress, promise and challenges. Nat Rev Immunol 2012, 12:786-798 